\title{
Architecture for the Secret-Key BC3 Cryptography Algorithm
}

\author{
Arif Sasongko $^{1}$, Hidayat ${ }^{2}$, Yusuf Kurniawan ${ }^{1} \&$ Sarwono Sutikno $^{1}$ \\ ${ }^{1}$ Electrical Engineering, School of Electrical Engineering and Informatics, \\ Institut Teknologi Bandung, Bandung, Indonesia \\ ${ }^{2}$ Computer Engineering, UNIKOM, Bandung, Indonesia \\ Email: ${ }^{1}$ asasongko,yusufk,ssarwono@ stei.itb.ac.id, ${ }^{2}$ hidayat@unikom.ac.id
}

\begin{abstract}
Cryptography is a very important aspect in data security. The focus of research in this field is shifting from merely security aspect to consider as well the implementation aspect. This paper aims to introduce BC3 algorithm with focus on its hardware implementation. It proposes an architecture for the hardware implementation for this algorithm. BC3 algorithm is a secret-key cryptography algorithm developed with two considerations: robustness and implementation efficiency. This algorithm has been implemented on software and has good performance compared to AES algorithm. $\mathrm{BC} 3$ is improvement of $\mathrm{BC} 2$ and AE cryptographic algorithm and it is expected to have the same level of robustness and to gain competitive advantages in the implementation aspect. The development of the architecture gives much attention on (1) resource sharing and (2) having single clock for each round. It exploits regularity of the algorithm. This architecture is then implemented on an FPGA. This implementation is three times smaller area than AES, but about five times faster. Furthermore, this BC3 hardware implementation has better performance compared to BC3 software both in key expansion stage and randomizing stage. For the future, the security of this implementation must be reviewed especially against side channel attack.
\end{abstract}

Keywords: BC3; FPGA; hardware implementation; symmetric key cryptography.

\section{$1 \quad$ Introduction}

Cryptography is one of main issue in protecting data security. It is a very important aspect in data security either at communication network or computer system. Encryption is implemented as the main method to preserve the security of electronic information [1]. This area of research has become very active. In the recent years, researchers look for cryptography algorithm with better implementation. The focus of the research is shifting from merely security aspect to consider as well the implementation aspect. Cryptographic algorithm whose characteristics: (1) mathematically secure/robust, (2) efficient and secure to implement is very desirable.

Received May, $2^{\text {nd }}, 2011$, Revised September $19^{\text {th }}, 2011$, Accepted for publication September, $19^{\text {th }}, 2011$. Copyright (C) 2011 Published by LPPM ITB, ISSN: 1978-3086, DOI: 10.5614/itbj.ict.2011.5.2.4 
The encryption algorithms are classified into two types: secret-key and publickey. The secret-key (symmetric key) algorithm uses same key for encryption and decryption so this key must be kept secret. It is shared exclusively between sender and receiver to protect the data in communication. Conversely, the public-key algorithm uses different key for encryption and decryption [2]. Secret key cryptography has more efficient implementation than public key cryptography. $\mathrm{BC} 3$ [3] is one of the secret-key algorithms [4]. This algorithm is result of enhancement of $\mathrm{BC} 2$ algorithm [5] and $\mathrm{AE} 3$ algorithm [6]. The security of $\mathrm{BC} 2$ and $\mathrm{AE} 3$ has been investigated quiet intensively. This new $\mathrm{BC} 3$ algorithm is modification of the $\mathrm{BC} 2$ algorithm especially for the reason of implementation efficiency. It is believed that the security aspect is not too different from $\mathrm{BC} 2$. It is developed by bearing in mind two considerations: projected security (robustness to attack) and efficient implementation. As most of secret key algorithm, for example AES, this algorithm can be divided into two stages: key expansion, and randomizing. The key expansion stage is performed to generate several subkeys from the main key (key master). The next stage is randomizing step wherein the data (plain text/chipper text) is manipulated using the subkeys to hide the information.

This algorithm has advantages compared to AES in two aspects. In the security aspect, the subkeys expansion in the BC3 are more secure than AES since the main key is extremely difficult to find even when the subkeys are found. In many cryptanalysis, for example differential and linear attacks, attacker try to find the subkeys before finding master key [7]. So without knowing the subkeys, attacker can not get master key. If the master key cannot be found from subkeys, attacker can never find the master key. The other advantage is that the key-expansion speed is much higher. The software implementation shows significant performance advantage compared to AES [5]. The hardware implementation of this algorithm is investigated in the work described in this paper.

The hardware implementation is very important from point of view performance and security, especially as countermeasure against timing attack[8], to avoid the key being left on the memory, and to save the computer memory. Therefore, we work to find architecture for $\mathrm{BC} 3$ hardware implementation which is efficient, especially number of gates or area since the speed of hardware implementation is certainly much higher than software implementation in several orders of magnitude.

This paper aims to introduce $\mathrm{BC} 3$ algorithm with focus on its hardware implementation. It proposes an architecture for hardware implementation of this algorithm. This architecture is described using VHDL and has been tested on Altera FPGA Cyclone® II type EP2C20F484C7 [9]. The result is compared 
with AES [4]. The security analysis is always the most important aspect in cryptography but it is not the subject of this particular paper. Moreover, it is believed that the security is not too difference from $\mathrm{BC} 2$, and it will be treated on other papers.

\section{BC3 Algorithm and the Software Implementation}

The $\mathrm{BC} 3$ algorithm is improvement of $\mathrm{BC} 2$ [5] and $\mathrm{AE} 3$ [10]. The characteristics of $\mathrm{BC} 3$ are quiet similar with $\mathrm{AES}$ and Camellia [11]. It is developed with two considerations: projected/measured securities/robustness and implementation efficiency. It means that this algorithm is designed to survive against various attacks or crypto analysis. At the same time, it is also designed to be implemented at various platforms efficiently and fast.

As described above, this algorithm can be divided into two stages: key expansion (or key schedule) and randomizing. Key expansion stage is performed to generate subkeys. The randomizing stage is performed to manipulate the data using the subkeys. The randomizing stage makes the plaintext encrypted or the cipher text decrypted. This randomizing stage consists of 11 rounds. In every round, a function $\mathrm{F}$ is applied to the data (plain text, chipper text or intermediate data produced by the previous round). This function uses different subkeys to manipulate the plain text in each round. This function is extremely difficult to be inverted without knowing the subkeys. The detail of this function is described later in Section 2.1.

The main features of BC3 algorithm are:

1. The width of input and output block is 64 bits (plain text and chipper text).

2. The main key length is 128 bits.

3. Key expansion is done in two steps (by six regular rounds and several logic operations). Regular round is the same $\mathrm{F}$ function used in the randomizing stage.

4. Randomizing stages in encryption and decryption processes are applied in eleven regular rounds. Each regular round consists of the same $F$ function.

5. In addition to the regular round, special function FA and $\mathrm{FA}^{-1}$ are used in every encryption and decryption process.

The key expansion stage is performed earlier to provide the subkeys for the randomizing stage, but for the presentation in this paper, the randomizing stage is described first. 


\section{Encryption and Decryption (Randomizing) Stage}

BC3 uses Feistel Network [2], so it encrypts and decrypts data with the same algorithm. This Feistel network is used in many algorithms such as DES, BLOWFISH, CAMELIA and Lucifer. Figure 1 shows the randomizing stage of encryption and decryption in $\mathrm{BC} 3$ algorithm. It uses eleven $\mathrm{F}$ functions and two special functions called FA and $\mathrm{FA}^{-1}$ functions. In each round, the key which is used to compute the $\mathrm{F}$ functions is different. These keys are called $\mathrm{K}_{1}$ for $1^{\text {st }}$ round, $\mathrm{K}_{2}$ for the $2^{\text {nd }}$ round, $\mathrm{K}_{3}$ for the $3^{\text {rd }}$ round, $\mathrm{K}_{4}, \mathrm{~K}_{5} \ldots \mathrm{K}_{11}$. The FA and $\mathrm{FA}^{-1}$ functions are inserted before the $5^{\text {th }}$ round and $8^{\text {th }}$ round respectively. Moreover, subkeys: KW1, KW2, KW3, and KW4 are XORed with the data before the first round and after the last round. All of these subkeys are previously derived from the main key in the key expansion stages.
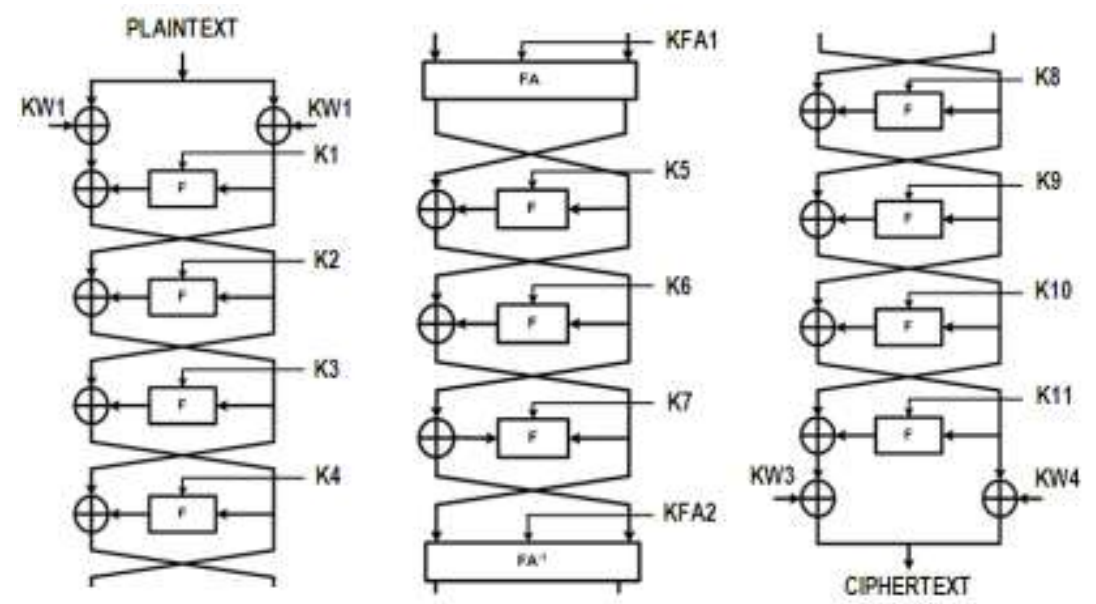

Figure 1 Randomizing stage of $\mathrm{BC} 3$ algorithm.

\section{The F Function}

The $\mathrm{F}$ function has two input. Each of them is 32 bits. This first input is either first half part of plaintext (in the first round of encripting process), half part of ciphertext (in the first round of decripting process), or data/output from previous round/function. The second input is a subkey. The first input is represented by $x$ with 32-bits, the second input is $\mathrm{K}$ and output is represented by $y$ with 32-bits in the following formula:

$$
\mathrm{y}=\mathrm{P}(\mathrm{S}[\mathrm{x}]) \oplus \mathrm{K}
$$

where:

$\oplus$ is an XOR logic operator 
$\mathrm{P}$ is operation of polynomial matrix multiplication;

$\mathrm{S}$ is operation of subtitution to $\mathrm{x}_{0}, \mathrm{x}_{1}, \mathrm{x}_{2}, \mathrm{x}_{3}$ inputs. $\mathrm{S}$ operation is done to every byte inputs;

$\mathrm{K}$ is sub keys $\mathrm{k}_{0}, \mathrm{k}_{1}, \mathrm{k}_{2}, \mathrm{k}_{3}$.

\section{Substitution Box (s-box)}

The $S$ box is used in the $S$ operation (subtitution). In this operation, each byte in the input vector is replaced by the byte from the S-box. S-box uses data in lookup table for substitution. The S-box of BC3 use a function that has properties almost similar to inversion function $\mathrm{f}(\mathrm{x})=\mathrm{x}^{-1}$ in finite field $F_{2}{ }^{8}$. The linear/affine operation was added to this function to block algebraic attack [12]. This inversion function is chosen because it has excellent properties to hamper linear and differential attack. Following data S-box (in hexadesimal):

\begin{tabular}{|c|c|c|c|c|c|c|c|c|c|c|c|c|c|c|c|}
\hline & B & $\mathrm{E}$ & $\mathrm{F}$ & 2 & 8 & F7 & $1 \mathrm{~F}$ & & & 26 & & & & & \\
\hline & 94 & $5 \mathrm{E}$ & $\mathrm{EE}$ & 78 & 91 & $7 \mathrm{~A}$ & $3 \mathrm{C}$ & & & & & & 97 & & \\
\hline & FC & $5 \mathrm{~F}$ & $\mathrm{AD}$ & 40 & $2 B$ & & & & & & & & & & \\
\hline & BA & $\mathrm{E}$ & 0 & $\mathrm{C} 0$ & D5 & 9 & C3 & & & & & $\mathrm{E}$ & U & DB & \\
\hline & $\mathrm{CD}$ & & & & & & & & & & & & & & \\
\hline & D4 & 99 & 0 & & 28 & (3) & נה & & & & & & & & \\
\hline & 3 & $\mathrm{C} 1$ & $\mathrm{C}$ & & & & & & & & & & & & \\
\hline & F3 & C6 & 8 & $3 \mathrm{~F}$ & & E5 & $\mathrm{OE}$ & & DC & & & & & & \\
\hline & $\mathrm{CC}$ & $6 B$ & & & & 98 & & & & & & & & & \\
\hline & 57 & E1 & $\mathrm{EB}$ & $8 \mathrm{~A}$ & & E0 & 5 & & 24 & & & & & & \\
\hline & $2 \mathrm{~F}$ & 40 & 74 & $\mathrm{CB}$ & 70 & 55 & $2 \mathrm{D}$ & & $\mathrm{AA}$ & & & & $E C$ & & \\
\hline & $8 \mathrm{C}$ & E2 & D6 & 12 & $\mathrm{E}$ & 10 & A & A & D2 & & 9 & & & & \\
\hline & 79 & $1 \mathrm{~A}$ & B5 & 2 & 18 & B7 & & & $4 \mathrm{~F}$ & & & & & & \\
\hline & $\mathrm{BF}$ & 44 & $3 \mathrm{~A}$ & C7 & 41 & $\mathrm{BE}$ & D1 & 15 & FA & & & & 00 & & \\
\hline & $\mathrm{AE}$ & $4 B$ & $7 E$ & C9 & $\mathrm{FE}$ & 85 & & & & & & & & & \\
\hline & A6 & $3 F$ & 30 & 20 & 63 & 72 & $\mathrm{~F} 2$ & A7 & $2 \mathrm{~A}$ & F9 & 61 & 32 & $6 \mathrm{D}$ & B9 & \\
\hline
\end{tabular}

The followings are examples how the s-box is used: if s-box input is 00 then it's output is $\mathrm{BB}_{\mathrm{x}}$, if s-box input is 01 then it's output is $8 \mathrm{~B}_{\mathrm{x}}$, etc.

\section{FA and FA $^{-1}$ Function}

The other functions used in the randomizing stages are FA and $\mathrm{FA}^{-1}$. FA function is applied before the $\mathrm{F}$ function in the $5^{\text {th }}$ round, $\mathrm{FA}^{-1}$ function is applied before the F function in the $8^{\text {th }}$ round. FA function is defined as follow:

$$
\begin{aligned}
& \mathrm{Y}_{\mathrm{R}}=\left(\left(\mathrm{X}_{\mathrm{L}} \cup \mathrm{KFA}_{1}\right)<<_{1}\right) \oplus \mathrm{X}_{\mathrm{R}} \\
& \mathrm{Y}_{\mathrm{L}}=\mathrm{X}_{\mathrm{L}} \oplus\left(\left(\mathrm{YR} \gg>_{1}\right) \cap \mathrm{KFA}_{2}\right)
\end{aligned}
$$


where:

$\gg>_{1}$ is shifts right 1 bit

$\ll<<_{1}$ is shifts left 1 bit

$\cap$ is AND logic operator/intersection

$\cup$ is OR logic operator/union

$\mathrm{FA}^{-1}$ is invers of FA. So, it is defined as:

$$
\begin{aligned}
& \mathrm{X}_{\mathrm{L}}=\mathrm{Y}_{\mathrm{L}} \oplus\left(\left(\mathrm{Y}_{\mathrm{R}} \gg>_{1}\right) \cap \mathrm{KFA}_{2}\right) \\
& \mathrm{X}_{\mathrm{R}}=\mathrm{Y}_{\mathrm{R}} \oplus\left(\left(\mathrm{X}_{\mathrm{L}} \cup \mathrm{KFA}_{1}\right)<<<_{1}\right)
\end{aligned}
$$

Figure 2 illustrates FA and $\mathrm{FA}^{-1}$ functions.
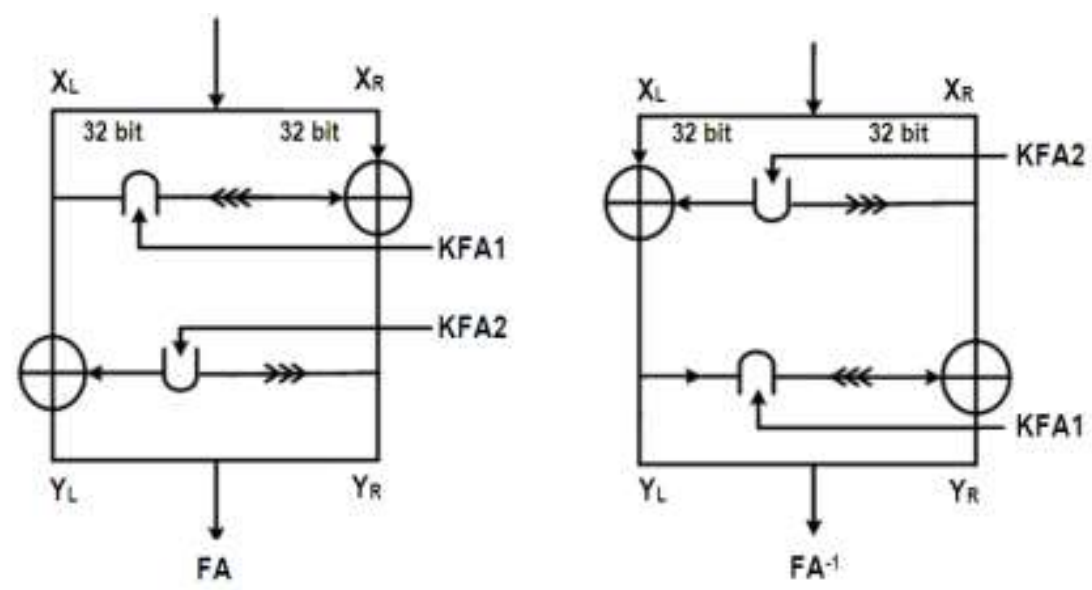

Figure 2 Special functions: $\mathrm{FA}$ and $\mathrm{FA}^{-1}$.

\section{$7 \quad$ BC3 Key Expansion}

BC3 key expansion or key-schedule process is used to derive the subkeys from the main key. Hence, this stage is performed before the randomizing stage. The characteristics of BC3 key schedule are: one way function, fast, having good diffusion and confusion properties. The process of key expansion is performed in two steps. First step is shown on Figure 3. The width of BC3 main key (K) is 128-bits. It can be regarded as concatenation of $\mathrm{K} 1$ and $\mathrm{K} 2(\mathrm{~K}=\mathrm{K} 1 \| \mathrm{K} 2)$. The widths of each key $\mathrm{K} 1$ and $\mathrm{K} 2$ are 64 -bits. The first 32 bit of $\mathrm{K} 1$ called $\mathrm{K} 1_{\mathrm{L}}$, the last 32 bit of $K 1$ called $K 1_{R}$. So is $K 2$, it is decomposed to $K 2_{L}$, and $K 2_{R}$. It should be noted that $\mathrm{K} 1$ and $\mathrm{K} 2$ are different from the subkeys $\mathrm{K}_{1}$, and $\mathrm{K}_{2}$. This 
main key $\mathrm{K}$ is used to generate $\mathrm{KA}, \mathrm{KB}, \mathrm{KC}$ and $\mathrm{KD}$ using process shown on Figure 3. The $\mathrm{F}$ function in this process is the same as $\mathrm{F}$ function in the randomizing stage. $\mathrm{C} 1, \mathrm{C} 2, \mathrm{C} 3, \mathrm{C} 4, \mathrm{C} 5, \mathrm{C} 6$ are constants which are parts of $\mathrm{BC} 3$ algorithm. These constants are described later in this section.
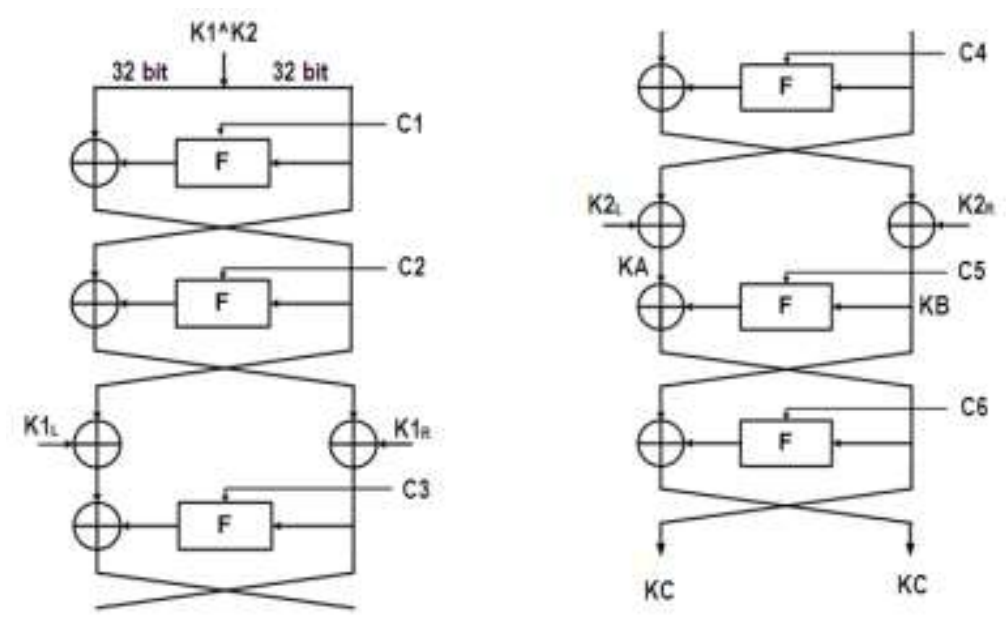

Figure 3 Initial part of BC3 key schedule.

After first step of BC3 key-schedule is done, second step is performed to generate the subkeys: $\mathrm{KW}_{1}, \mathrm{KW}_{2}, \mathrm{KW}_{3}, \mathrm{KW}_{4}, \mathrm{~K}_{1}, \mathrm{~K}_{2}, \mathrm{~K}_{3}, \mathrm{~K}_{4}, \mathrm{~K}_{5}, \mathrm{~K}_{6}, \mathrm{~K}_{7}, \mathrm{~K}_{8}$, $\mathrm{K}_{9}, \mathrm{~K}_{10}, \mathrm{~K}_{11}, \mathrm{KFA}_{1}, \mathrm{KFA}_{2}$. The computation is described as follow:

$$
\begin{aligned}
& \mathrm{KE}=\mathrm{KA} \cap \mathrm{KB} \oplus \mathrm{KC} \\
& \mathrm{KF}=\mathrm{KA} \cup \mathrm{KB} \oplus \mathrm{KD} \\
& \mathrm{KG}=\mathrm{KF} \cap \mathrm{KE} \oplus \mathrm{KA}
\end{aligned}
$$

Then, the following computation is performed:

$$
\begin{array}{ll}
\mathrm{KW}_{1}=\mathrm{KE} \cup \mathrm{KF} \oplus \mathrm{KG} & \mathrm{K}_{7}=\mathrm{K}_{6} \cup \mathrm{KW}_{2} \oplus \mathrm{KFA}_{1} \\
\mathrm{KW}_{2}=\mathrm{KF} \cap \mathrm{KG} \oplus \mathrm{KD} & \mathrm{KFA}_{2}=\mathrm{K}_{1} \cup \mathrm{K}_{5} \oplus \mathrm{K}_{6} \\
\mathrm{~K}_{1}=\mathrm{KE} \cap \mathrm{KW}_{2} \oplus \mathrm{KF} & \mathrm{K}_{8}=\mathrm{C}_{3} \cup \mathrm{K}_{3} \oplus \mathrm{K}_{7} \\
\mathrm{~K}_{2}=\mathrm{KE} \cup \mathrm{KF} \oplus \mathrm{KW}_{1} & \mathrm{~K}_{9}=\left(\mathrm{K}_{5} \gg>>_{1}\right) \oplus \mathrm{K}_{3} \\
\mathrm{~K}_{3}=\mathrm{KW}_{1} \cap \mathrm{KW}_{2} \oplus \mathrm{K}_{2} & \mathrm{~K}_{10}=\mathrm{K}_{8} \cup \mathrm{K}_{4} \oplus \mathrm{K}_{5} \\
\mathrm{~K}_{4}=\mathrm{K}_{1} \cap \mathrm{KW}_{2} \oplus \mathrm{K}_{3} & \mathrm{~K}_{11}=\mathrm{K}_{3} \cup \mathrm{K}_{5} \oplus \mathrm{K}_{6} \\
\mathrm{KFA}_{1}=\mathrm{C}_{1} \cup \mathrm{KE} \oplus \mathrm{K}_{4} & \mathrm{KW}_{3}=\mathrm{K}_{9} \cup \mathrm{K}_{4} \oplus \mathrm{K}_{6} \\
\mathrm{~K}_{5}=\mathrm{C}_{2} \cup \mathrm{KW}_{2} \oplus \mathrm{K}_{3} & \mathrm{KW}_{4}=\mathrm{K}_{2} \cup \mathrm{K}_{8} \oplus \mathrm{K}_{9}
\end{array}
$$




$$
\mathrm{K}_{6}=\mathrm{K}_{1} \cap \mathrm{K}_{2} \oplus \mathrm{K}_{5}
$$

The constants: $\mathrm{C} 1, \mathrm{C} 2, \mathrm{C} 3, \mathrm{C} 4, \mathrm{C} 5$ and $\mathrm{C} 6$ have the same size 32 bits. The constants $\mathrm{C} 1$ and $\mathrm{C} 2$ are taken from $\sqrt{ } 0.7$ (after the zero point ....) which is D62F 59FB D597 BEF1 (64 bit). The constants C3 and C4 are taken from $\sqrt{ } 0.8$ (after the zero point ....) which is E4F9 2E2D FF6E C9AB. The constants $\mathrm{C} 3$ and $\mathrm{C} 4$ are taken from $\sqrt{ } 0.9$ (after the zero point ....) which is F2DC E89B 636C B246. So the constant are:

$$
\begin{aligned}
& \mathrm{C} 1=\mathrm{D} 62 \text { F59FBx } ; \mathrm{C} 2=\mathrm{D} 597 \mathrm{BEF} 1 \mathrm{x} \\
& \mathrm{C} 3=\mathrm{E} 4 \mathrm{~F} 92 \mathrm{E} 2 \mathrm{Dx} ; \mathrm{C} 4=\text { FF6EC9ABx } \\
& \mathrm{C} 5=\text { F2DCE89Bx } ; \mathrm{C} 6=636 \mathrm{CB} 246 \mathrm{x}
\end{aligned}
$$

The objectives of the utilization of these constants are to get random keys and to show that there is not any backdoor on this algorithm.

\section{BC3 Software Implementation}

The source code of BC3 software implementation is written in C language [3]. This algorithm is constructed in 1150 lines code. The key schedule stage and randomizing stage take 0.7731 us and 0.71865 us respectively on PC with 1.2 $\mathrm{GHz}$ AMD Duron Processor. This result is better than AES [5]. Figure 4 shows the data flow diagram of $\mathrm{BC} 3$ software.

This implementation is very fast because it does not have any multiple rotations in program. Multiple rotations of data can slow down the software implementation significantly. The implementation consists of Boolean logic, substitution, and single bit rotation. This software is developed in the way that can be ported in different platform, processor and OS. It is also easy to compare this implementation with CAMELIA [13] or AES [14].

The Table 1 shows comparison of $\mathrm{BC} 3$ and some other popular algorithm. This comparison is using Intel Celeron $1,3 \mathrm{Ghz}$ with $512 \mathrm{MB}$ memory. All software is compiled using ANSI C.It must be noted that BC3 has 64 bits input/output meanwhile AES and Camellia have 32 bit input/output. To get a fair comparison, the the smallest measured data unit is 128 bit block. This data unit is encrypted once using AES or Camellia and encrypted twice using BC3. So, 128 millions bit is encrypted in one million process using CAMELLIA and AES or 2 million process using $\mathrm{BC} 3$. 


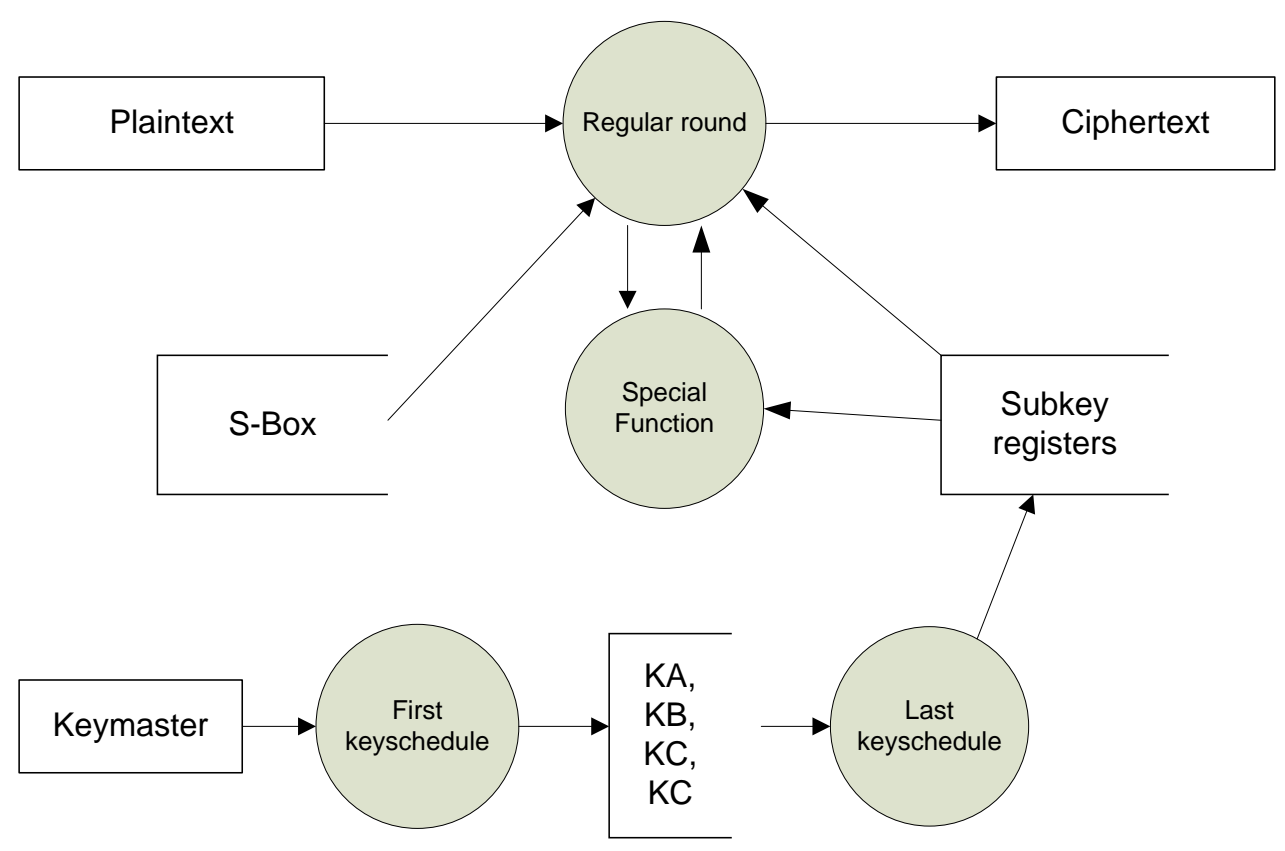

Figure 4 The $1^{\text {st }}$ level DFD of BC3 software.

The case is almost similar for key expansion. One key expansion process for AES or Camellia is equivalent to two key expansions for $\mathrm{BC} 3$. The number on the figure has been multiplied by two for the BC3.

Table 1 Comparison of Software Implementation.

\begin{tabular}{lcc}
\hline \multicolumn{1}{c}{ Algorithm } & $\begin{array}{c}\text { Encryption Time for 128 } \\
\text { millions bit (in second) }\end{array}$ & Key expansion time \\
\hline AES & 0.453 & 1.063 \\
BC2 & 0.765 & 0.378 \\
BC3 & 0.485 & 0.390 \\
Camellia & 1.187 & 1.172 \\
Khazad & 0.687 & 1.063 \\
IDEA & 2.235 & 1.523 \\
\hline
\end{tabular}

The AES has slow key schedule because encryption setup process is different from decryption setup process. If CFB encryption mode is used, then AES Key expansion is faster since it doesn't need decryption and key expansion for decryption. 


\section{BC3 Hardware Implementation}

The objective of this research is to propose $\mathrm{BC} 3$ architecture with very efficient area. The implementation with minimum area can be easily used as coprocessor, as part of smartcard, or other embedded system, wherein this algorithm is typically used. It is targeted that for every block of data (64 bit), the hardware being designed must need less than 12 clocks to perform encryption/decryption. Thus, $200 \mathrm{MHz}$ hardware implementation can have throughput up to1 Gbps. So, it will be able to be used in Gigabit Ethernet or T1 network.

On the BC3 algorithm, the same F function is used every round in both randomizing stage and first part of key expansion stage. Thus, it is very advantageous to have a single block capable to perform this function multiple times. In addition, it is very efficient also if this block can performed FA and FA $^{-1}$ Function. For this reason, we design this computing block to perform those functions as efficient and flexible as possible and it is named Regular Round block. This block must be a combinational digital circuit. Therefore every round needs only one clock. This choice must be taken to ensure that 64 bit input can be treated in less than 12 clocks as the objective described above.

The other necessary computing block is a block to calculate the subkeys in the second part of key expansion: $\mathrm{KW}_{1}, \mathrm{KW}_{2}, \mathrm{KW}_{3}, \mathrm{KW}_{4}, \mathrm{~K}_{1}, \mathrm{~K}_{2}, \mathrm{~K}_{3}, \mathrm{~K}_{4}, \mathrm{~K}_{5}, \mathrm{~K}_{6}$, $\mathrm{K}_{7}, \mathrm{~K}_{8}, \mathrm{~K}_{9}, \mathrm{~K}_{10}, \mathrm{~K}_{11}, \mathrm{KFA}_{1}, \mathrm{KFA}_{2}$. This block takes $\mathrm{Ka}, \mathrm{Kb}, \mathrm{Kc}$, and $\mathrm{Kd}$ as inputs and it is called Keyschedule block. Hence, the design has two computing blocks: regular round block and key schedule block. The regular round block is used in the randomizing and key scheduling stage. The key schedule block is used in the keyscheduling stage only.

Besides the computing blocks, it is necessary to have control blocks and a register block. The register block (or memory) is needed to store the subkeys. It is then called subkey registers. The control box is an FSM (finite state machine) that controls the computing block to perform their functions. So there are three control blocks: FSM key scheduling, FSM Encryption, and FSM decryption. In addition to those control blocks, it is necessary to have a main control FSM to manage the input output process and to coordinate the three other control FSMs. As depicted on Figure 6, the proposed architecture has four control/FSM blocks (Main Controller, FSM Keyschedule, FSM Encryption, FSM Decryption), two computing blocks (Regular Round, Keyschedule) and a register file/memory (Subkey register). This architecture is depicted in Figure 5. 


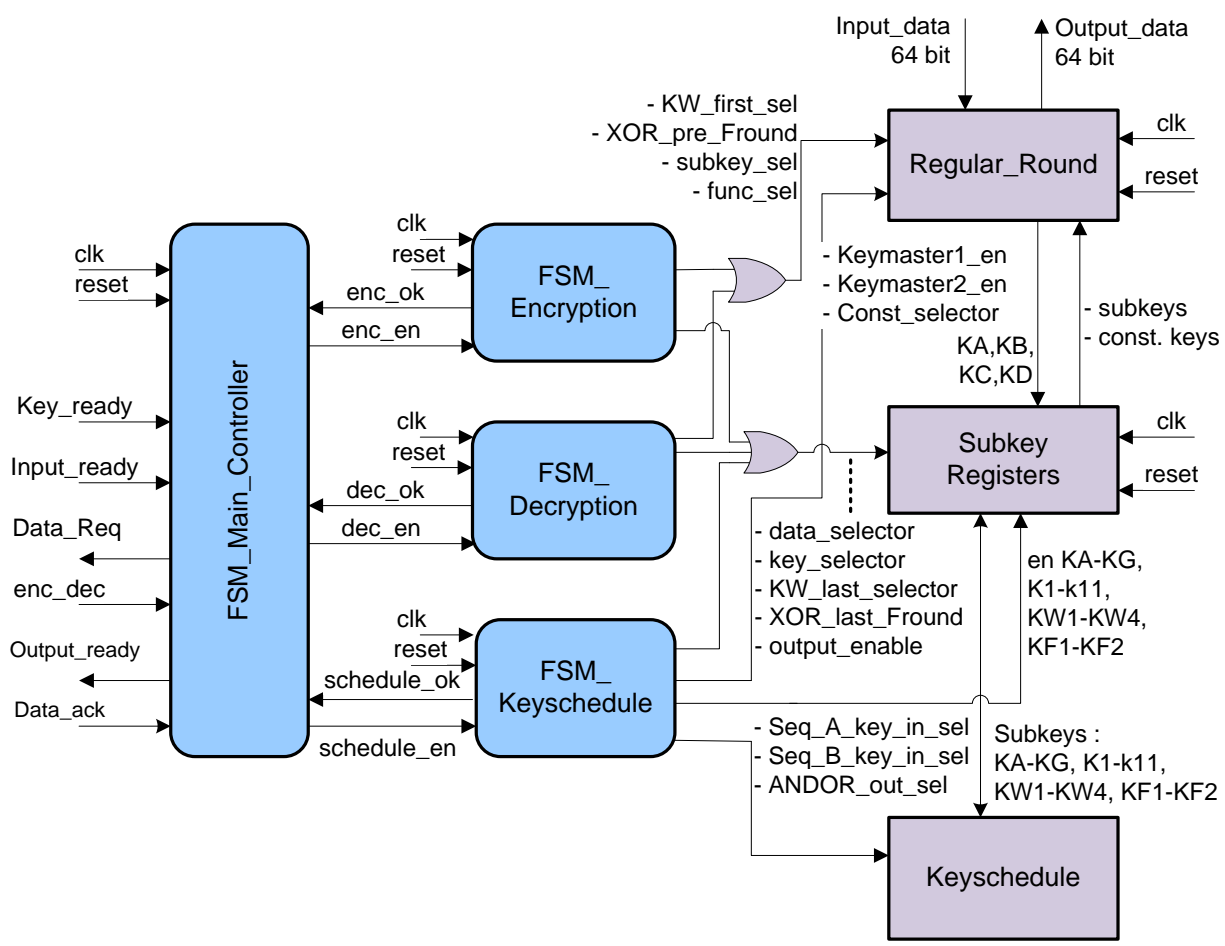

Figure 5 BC3 algorithm architecture.

\section{Control Block/FSM}

The architecture above can be used for encryption and decryption. The operation of this architecture is defined in the FSM. For encryption process, the signal enc_dec must be driven high by the user (to set the mode to encryption). The operation of this architecture in this mode is as follow:

1. The main key must be entered through the input data port. The key ready signal must be driven high during this process to inform the main controller that a new key is fed to the system. Then, the main controller is sending signal (schedule enable) to the FSM_Keyschedule so the FSM_Keyschedule takes the control of the subkey registers block and the two computing blocks.

2. The FSM_Keyschedule controls the regular round block and uses it to produce $K_{A}, K_{B}, K_{C}$, and $K_{D}$. The results are then stored in the register file (the subkey register block).

3. The FSM_Keyschedule controls the Keyschedule block and uses it to produce the subkeys by using $\mathrm{K}_{\mathrm{A}}, \mathrm{K}_{\mathrm{B}}, \mathrm{K}_{\mathrm{C}}$, and $\mathrm{K}_{\mathrm{D}}$ as input. The result is 
then stored in the subkey registers block. The FSM_Keyschedule is then give the control back to FSM Main controller through the signal Schedule_ok. This signal informs the main controller that the key expansion process is completed.

4. The main controller sends the signal Data Req to tell the user that the system is ready to receive the data/plain text

5. When the user needs to encrypt data, the user must put the data to input_data port and drives the signal Input_Ready high.

6. The main controller sends the signal Enc_Enable to tell the FSM Encryption to starti its operation.

7. The Encryption FSM uses the regular round block to encrypt the data. It uses the proper subkeys which are stored in the Subkey registers as key in every round.

8. The output of encryption is then sent to Output_Data port and the control is given back to FSM main controller.

9. The FSM Main controller sends the signal Output ready to the user.

10. The user can take the result and sends acknowledge to the system

11. The main FSM sends signal Input_Ready, so the user can put the next data to be encrypted. The operation is loop back to step (4).

For the decryption mode, the operation is almost similar to decryption mode. The difference is that, at step 6, the main FSM send signal Dec_Enable to FSM Decryption instead of sending Enc_Enable to the FSM Encryption. Then, the FSM Encryption is taken the control of the Regular Round block. The signal enc_dec must be driven low by the user (to set the mode to decryption).

\section{Regular_round Block}

Regular_round block is used for round process in randomizing stage and first part of key-scheduling stage. Figure 7 shown the regular_round block. Basically, this block can be divided into two parts: input selector and $\mathrm{F}$ function. The input selector feed the block with the proper the input for each particular round. There are four possibilities of input: (1) from input port in the first round, (2) from previous round directly, (3) from previous round trough FA function, or (4) from previous round trough $\mathrm{FA}^{-1}$ function. Figure 6 shows the detail of this block.

The F function in the Regular Round Block can take various key depending on various subkey selectors. The FSM_Encryption/FSM_Decryption (controller) drives these subkey selectors. The subkeys are stored and restored on the subkey registers. This Regular Round designed in order to process one round per cycle. So, this architecture needs eleven cycles to execute eleven rounds in encryption/decryption process. 


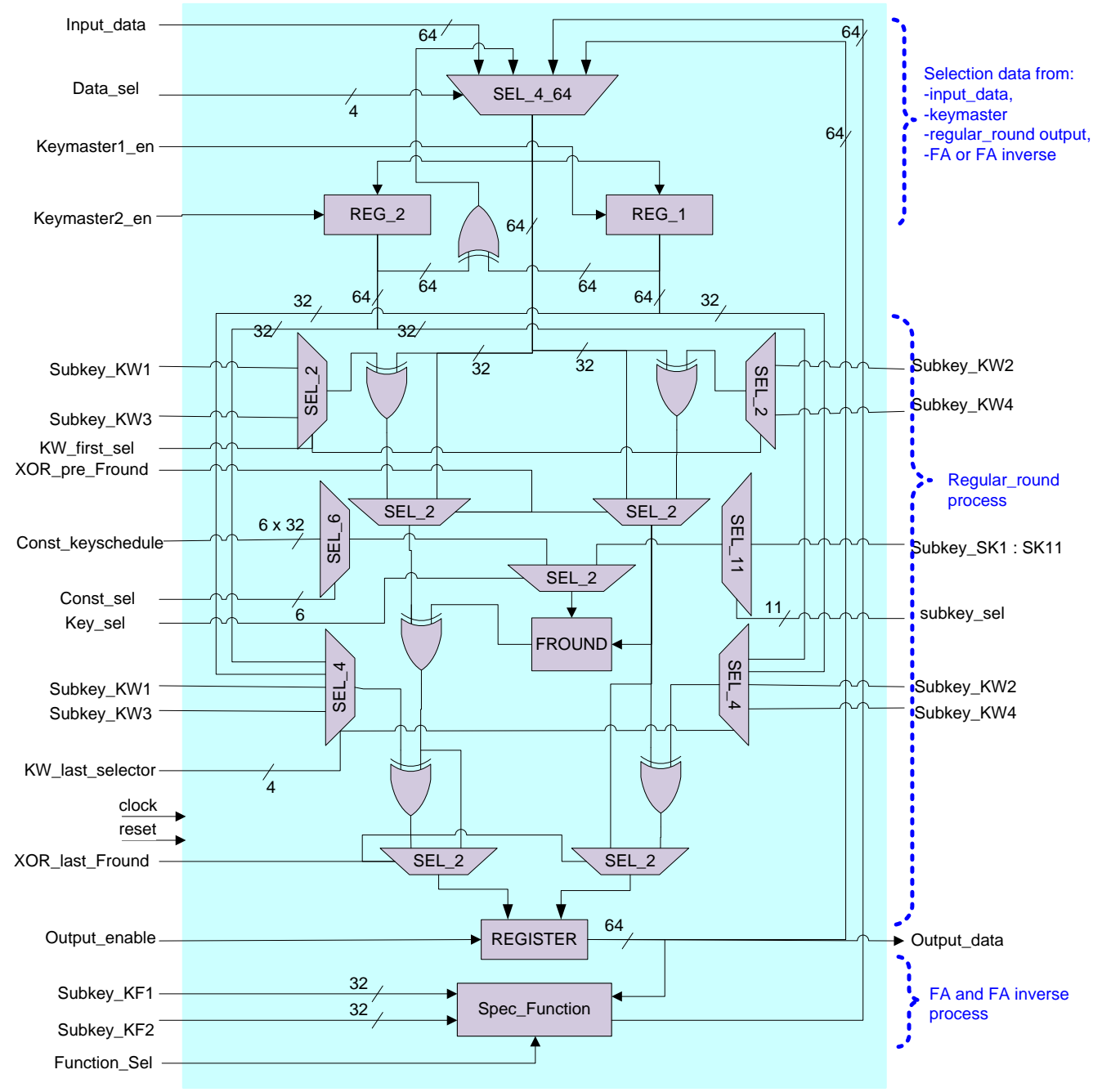

Figure 6 The regular_round block.

\section{Key-schedule Block}

Key-schedule block is design to perform the second part of key expansion process. It takes $\mathrm{KA}, \mathrm{KB}, \mathrm{KC}, \mathrm{KD}$ as input and generates the subkeys. Figure 7 shown the keyschedule block. It consists of various combinational block such as AND gate, XOR gate, OR gate, rotation, and combination of them. Moreover, there are several multiplexer for selecting and channeling the data. 


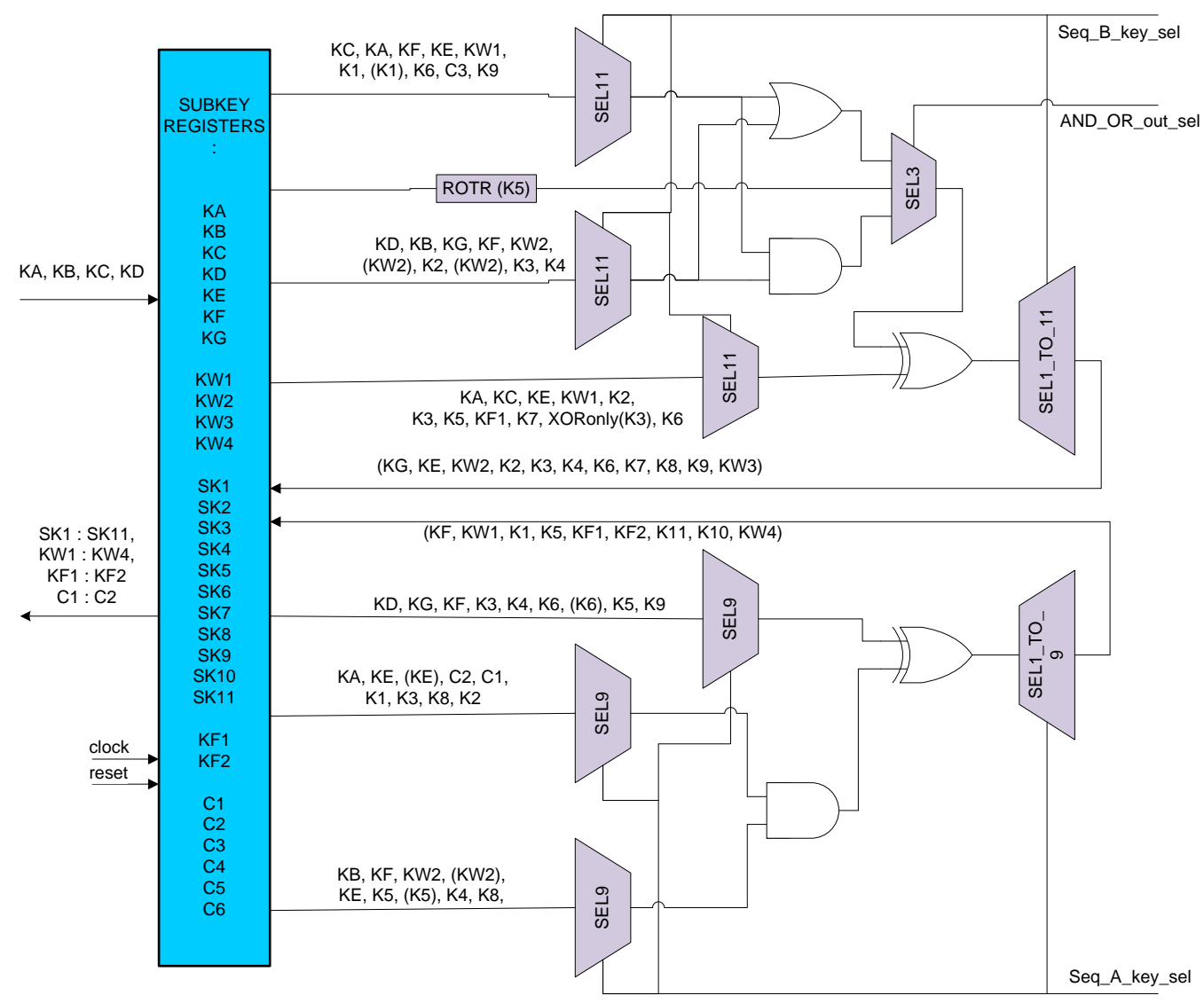

Figure 7 The keyschedule block.

\section{Result of BC3 Hardware Implementation}

The BC3 design is described in register transfer level by using VHDL. The number of files written for this design is 29 files. The design is then simulated using test vector. It is then compared with the software implementation and manual calculation to ensure the correctness.

The design is then implemented using Altera FPGA Cyclone ${ }^{\circledR}$ II type EP2C20F484C7 [9]. The result of synthesis shows that hardware design of BC3 algorithm requires 3098 logic elements, 1119 registers and $136 \mathrm{I} / \mathrm{O}$ pins. The timing report informs estimation critical path along $32.620 \mathrm{~ns}$ or $30.66 \mathrm{MHz}$. This indicates that fastest value which can be passed to this design is 30.66 $\mathrm{MHz}$ or fastest period time is $32.620 \mathrm{~ns}$. The Cyclone II is a low cost/low performance FPGA from Altera. Obviously, this speed can be improved using 
faster FPGA or ASIC. The ASIC implementation can be faster in some orders of magnitude.

The comparisons of BC3 hardware with BC3 software is shown on Table 2.

Table 2 Comparison of BC3 implementation.

\begin{tabular}{lll}
\hline Criteria & $\begin{array}{l}\text { BC3 hardware } \\
\text { (assume using fastest } \\
\text { period time (30.620ns)) }\end{array}$ & $\begin{array}{l}\text { BC3 software } \\
\text { (on AMD Duron } \\
\text { Processor 1.2 GHz) }\end{array}$ \\
\hline $\begin{array}{l}\text { Key expansion process } \\
\text { (encryption/decryption) }\end{array}$ & 0.55454 us & $0.7731 \mathrm{us}$ \\
Randomizing process & 0.35882 us & 0.71865 us \\
\hline
\end{tabular}

Table 2 show that key-scheduling and encryption/decryption processes on hardware is 0.55454 us and 0.35882 us, respectively. The key-scheduling and encryption/decryption processes on software are 0.7731 us and 0.71865 us, respectively. So, the time for key-scheduling and encryption/decryption processes on hardware implementation is faster than on previous software implementation.

The comparison of result BC3 hardware implementation with AES hardware [4] is shown on Table 3.

Table 3 Comparison of $\mathrm{BC} 3$ and AES.

\begin{tabular}{lll}
\hline Criteria & BC3 (128 bits) & AES (128 bits) \\
\hline Logic elements & 3,098 & 10,338 \\
$\begin{array}{l}\text { Randomizing process } \\
\text { (encryption/decryption) } \\
\text { Key expansion }\end{array}$ & 11 clock cycles & 54 clock cycles \\
& 17 clock cycles & 10 clock cycles \\
\hline
\end{tabular}

The result of area of $\mathrm{BC} 3$ algorithm is smaller than area of AES [4]. The main advantages come from: (1) flexible regular_round block so each round can be executed in single clock, (2) sharing the computation block when possible, (3) the design of $\mathrm{BC} 3$ algorithm which is very regular/homogenous. Actually, the ideas used in this architecture can be also to construct an architecture for AES.

\section{Conclusions}

This paper presents an architecture for implementing BC3 algorithm on hardware. This implementation needs fewer logic elements than AES [4] and has better performance. Furthermore, the BC3 hardware implementation has better performance compared to $\mathrm{BC} 3$ software both in key expansion and encryption/decryption. Therefore, this architecture is proven to result a low area 
implementation thanks to the exploitation of the regularity of the $\mathrm{BC} 3$ algorithm. Idea used in this architecture may be utilized also for other algorithm using Feistel network. For the future, the robustness of this implementation must be reviewed especially against side channel attack such as timing attack and power analysis attack.

\section{References}

[1] Goldreich, O., Foundations of Cryptography, Vol. I Basic Tools, Cambridge, 2006

[2] Stallings, W., Cryptography and Network Security Principles and Practices ( $4^{\text {th }}$ ed.). New Jersey: Prentice Hall, 2005.

[3] Kurniawan, Y., Algoritma Enkripsi Indonesia BC3, http://ysfk2008. wordpress.com, Accessed on November, 2011.

[4] Satoh, A., Morioka, S., Takano, K., \& Munetoh, S., A Compact Rijndael Hardware Architecture with S-Box Optimization, ASIACRYPT 2001, LNCS 2248. pp. 239-254, 2001.

[5] Kurniawan, Y., Suwandi, A., Mardianto, M. S., Supriana, I., Sutikno, S. The New Block Cihper: BC2, International Journal of Network Security, 8(1), pp. 16-24, 2009.

[6] Kurniawan, Y., Analisis Sandi Diferensial AE3, Proceeding of Seminar Nasional IC2007 BINUS University, Indonesia, 2007.

[7] Biryukov, A., Khovratovich, D. \& Nikolić, I. , Distinguisher and Related-Key Attack on the Full AES-256, Lecture Notes in Computer Science, 5677/2009, Springer-Verlag, 2009

[8] Kocher, Paul C., Timing Attacks on Implementations of Diffie-Hellman, RSA, DSS, and Other Systems, CRYPTO, 1996.

[9] ---, DBC2C20-Cyclone II Development Board, Altera, 2008

[10] Kurniawan, Y., Algoritma Enkripsi AE3 dan Analisis Sandi Linear, Proceeding of Konferensi Nasional Sistem Informasi, Indonesia, 2008.

[11] Matsui, M., Nakajimi, J., A Description of Camellia Encryption Algorithm, from http://www.ipa.go.jp/security/rfc/RFC3713EN.html, Accessed on Februari, 2009.

[12] Katz, J. \& Lindell, Y., Introduction to Modern Cryptography, Chapman \& Hall/CRC, 2008.

[13] Aoki, K., Ichikawa, T., Kanda, M., Matsui, M., Moriai, S., Nakajima, J. \& Tokita, T., Camellia : A 128-Bit Block Cipher Suitable for Multiple Platforms - Design and Analysis, Lecture Notes in Computer Science, 2012/2001, Springer-Verlag, 2001

[14] Bertoni, G., Breveglieri, L., Fragneto, P., Macchetti, M. \& Marchesin, S., Efficient Software Implementation of AES on 32-Bit Platforms, Lecture Notes in Computer Science, 2523/2003, Springer-Verlag, 2003. 